\title{
On the acoustic properties of parallel arrangement of multiple micro-perforated panel absorbers with different cavity depths
}

\author{
Chunqi Wang ${ }^{\text {a) }}$ and Lixi Huang \\ Department of Mechanical Engineering, The University of Hong Kong, Pokfulam Road, Hong Kong
}

(Received 13 September 2010; revised 30 April 2011; accepted 4 May 2011)

\begin{abstract}
The acoustic properties of a compound micro-perforated panel (MPP) absorber array are investigated. The absorber array consists of three parallel-arranged MPP absorbers with different cavity depths. A finite element procedure is used to simulate its acoustic behaviors under normal incidence. Experimental studies are carried out to verify the numerical simulations. Due to different reactance matching conditions in the absorber array, strong local resonance occurs and the corresponding local resonance absorption dominates. Compared with single MPP absorber, the absorber array requires lower acoustic resistance for good absorption performance, and the resonance frequencies shift due to inter-resonator interactions. The different acoustic resistance requirement is explained by considering the reduced effective perforation rate of the MPP in the absorber array. The performance of the absorber array varies with the sizes and spatial arrangement of the component absorbers. When the distance between component absorbers is larger than a quarterwavelength, the above-mentioned parallel absorption mechanism diminishes. In the experimental study, the normal incidence absorption coefficients of a prototype MPP absorber array are tested. The measured results compare well with the numerical predictions. The experimental study also shows that although other absorption mechanisms may exist, dissipation by the MPP is dominant in the MPP absorber array. (C) 2011 Acoustical Society of America. [DOI: 10.1121/1.3596459]
\end{abstract}

PACS number(s): 43.55.Ev, 43.50.Gf [KVH]

Pages: 208-218

\section{INTRODUCTION}

As an innovative fiber-free solution to the noise control problem, the micro-perforated panel (MPP) is finding more and more applications in areas such as room acoustics, ${ }^{1,2}$ environmental noise abatement ${ }^{3}$ and duct mufflers. ${ }^{4}$ An MPP is a thin flat plate perforated with numerous sub-millimeter orifices. By reducing the sizes to sub-millimeter scale, the perforations themselves can provide enough acoustic resistance and low acoustic reactance necessary for an absorber without the use of any porous material. ${ }^{5,6}$ The basic construction of an MPP absorber consists of an MPP fitted in front of a rigid backing wall, as illustrated in Fig. 1(a). The backing wall is usually parallel to the MPP with a constant air gap between them. The acoustic performance of such a device can be completely determined by the orifice diameter, the perforation rate, the panel thickness and the depth of the air gap. 6,7

Although the MPP offers an outstanding alternative to the traditional porous materials, its sound absorption capability is usually not quite enough for a general purpose absorber. For instance, the commonly used MPP with orifice diameter in the range of $0.5-1 \mathrm{~mm}$ is able to achieve a halfabsorption (i.e., the sound absorption coefficient $\alpha \geq 0.5$ ) bandwidth of one or two octaves. Both the bandwidth and the half-absorption criterion are insufficient to compete with the porous materials. Theoretically, the half-absorption bandwidth can be further extended up to three or four octaves if minute orifices of diameter $0.1-0.2 \mathrm{~mm}$ are used. ${ }^{7}$ However, the excess cost associated with the manufacturing

\footnotetext{
a) Author to whom correspondence should be addressed. Electronic mail: cqwang@hku.hk
}

process prevents the wide-spread use of them in the noise control industry. Another approach to broaden the absorption bandwidth is to adopt a double-layer MPP construction. ${ }^{6} \mathrm{By}$ adding a second MPP to the primary one in tandem, the double-layer design can extend the absorption bandwidth to lower frequencies, while at the cost of increased cavity depth due to the extra layer construction. Aiming for broadband absorption, a more straightforward approach is to arrange multiple MPP absorbers of different frequency characteristics in parallel, hence an MPP absorber array. It seems natural that broader absorption bandwidth can be achieved by combining different frequency bands together. However, so far, a thorough understanding of the acoustic properties of such an absorber array is still lacking, which has motivated the current investigation.

Before presenting the details of the present investigation, some related previous work is reviewed below. In an early study by Zha et al., ${ }^{8}$ a parallel arrangement of two MPP absorbers of different cavity depths was proposed for broadband performance, and its normal incidence absorption coefficients were tested experimentally using an impedance tube. Yairi et $a l .{ }^{9}$ later tested a similar parallel arrangement design and compared the measured results with predictions based on the equivalent electrical circuit model. ${ }^{5,6}$ Both studies showed that the parallel arrangement of two different MPP absorbers was able to obtain a broader absorption band. Moreover, the absorption performance of the absorber array could be better than the average of the two component MPP absorbers. In the latter work, experimental studies were also extended to measure the reverberation absorption coefficients of larger size prototypes in meters. In a recent numerical study, Sakagami et al. ${ }^{10}$ predicted the absorption performance of a parallel arrangement of 
two different MPP absorbers by considering the excess attenuation caused by the impedance discontinuity. The results are helpful for understanding the absorption characteristics of the MPP absorber arrays, though the validity of the numerical modeling is to be verified. To summarize, most of the existing work has to some extent demonstrated the potential of obtaining broader frequency band through combining different resonances. However, few efforts have been made to further investigate the distinct acoustic behaviors of the MPP absorber array and the physics behind them. The acoustic benefits of the parallel arrangement of multiple MPP absorbers have not been fully characterized either. Note that there are also investigations on the use of surface and volume heterogeneities to increase the absorption efficiency in some other sound-absorbing structures. For instance, Mechel $^{11}$ demonstrated numerically that broadband sound absorption could be achieved by covering a restive layer onto the front surface of a Schroeder diffuser, which consists of an array of wells with different depths arranged in a pseudo-random manner. The numerical findings were later verified experimentally by $\mathrm{Wu}$ et al $^{12}$ They also performed an optimization procedure to tune the depths sequence for better absorption performance. In another study by Groby et al., ${ }^{13}$ the acoustic properties of a finite layer of porous material in front of a periodic irregular grating were investigated. The enhanced sound absorption performance was mainly explained by the modified modes of the backed-layer and modes of the grating which are excited due to the periodic irregular grating.

The primary purpose of the present work is to investigate the acoustic properties of a parallel arrangement of multiple MPP absorbers with different cavity depths (i.e., the MPP absorber array), as shown in Fig. 1(b). Special attention is paid to the physical explanation of the absorption mechanisms within such an MPP absorber array. The results provide a useful basis for the design of MPP absorber array as well as a better understanding of its unique acoustic characteristics. In doing so, a reliable numerical model is required to describe properly the acoustic behaviors of the MPP

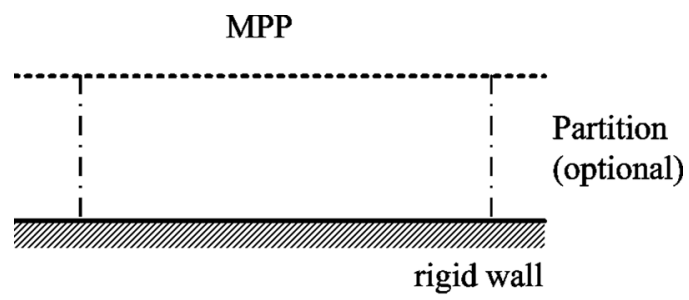

(a)

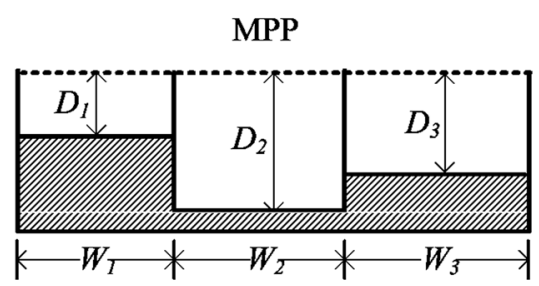

(b)

FIG. 1. Schematic diagrams of the MPP absorbers. (a) A single MPP absorber with uniform air gap. The partitioning wall is optional. (b) A compound MPP absorber array with three partitioned cavities. absorber array both for the theoretical investigation and for the practical design purposes. The equivalent electrical circuit analogy is easy to use to calculate the absorption coefficients, but the details of the acoustic field are not available for analysis. Moreover, as the electrical circuit model represents the extreme situation in which the MPP absorber array is infinitesimal, ${ }^{14}$ the effect of the finite geometry of the component MPP absorbers on the absorption performance is not captured. In this study, a finite element procedure is established and validated against experimental results and well-established analytical solutions. In what follows, Sec. II describes the finite element modeling procedure of the MPP absorber based on a two-dimensional (2D) configuration. The finite element simulation compares well with analytical solutions available in literatures. Section III investigates numerically the acoustic properties of MPP absorber arrays, which consists of three parallel-arranged MPP absorbers with different cavity depths. Section IV describes the experimental studies conducted to validate the finite element modeling procedure as well as the numerical findings.

\section{FINITE ELEMENT MODELING OF THE MPP ABSORBER}

A finite element procedure is established to simulate the acoustic behaviors of the MPP absorber under normal incidence. The modeling procedure is described below based on a simple 2D configuration of a single MPP absorber, which consists of a rectangular cavity (E-F-G-H) and an MPP covering on the open side of the cavity $(\mathrm{E}-\mathrm{H})$, as shown in Fig. 2. The MPP itself can be assumed to be either flexible or rigid, depending on the thickness and the material of the panel. Owing to the versatility of the finite element simulation, the backing cavity in Fig. 2 can be extended to more complicated shapes without any fundamental changes in the modeling procedure, for example, the trapezoidal-cavity backed MPP absorber reported earlier ${ }^{15}$ and the MPP absorber array in Fig. 1(b). Following the ISO standard ${ }^{16}$ on determination of sound absorption coefficient, a rectangular duct (a channel in the 2D model) is connected to the MPP absorber in the numerical model, representing the impedance tube in the experimental rig. Such a configuration is primarily for the simulation of the acoustic properties of the MPP absorber under normal incidence, which can be conveniently tested experimentally. The overall modeling procedure applies to the situation of oblique incidence, but a different treatment of the boundary conditions is required.
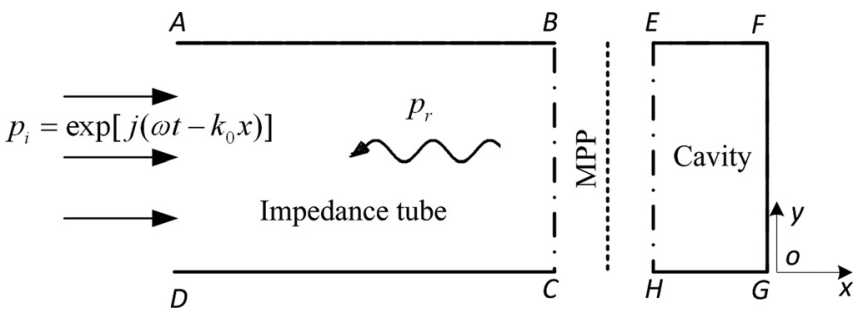

FIG. 2. Numerical model for the MPP absorber which consists of an MPP covering the open side $(\mathrm{E}-\mathrm{H})$ of a rectangular cavity. A virtual impedance tube is fitted to the MPP absorber at the open side. The walls of the impedance tube and the rectangular cavity are assumed to be acoustically rigid. 
Assume that a plane incident wave comes from the lefthand side of the duct with unit amplitude,

$$
p_{i}=\exp \left[j\left(\omega t-k_{0} x\right)\right]
$$

where $\omega=2 \pi f$ is the angular frequency, $k_{0}$ is the acoustic wavenumber, $j=\sqrt{-1}$ is the imaginary unit. When the acoustic wave $p_{i}$ is incident on the MPP, some of the acoustic energy is reflected, and the rest is absorbed by the MPP as well as the sound absorbing materials (if any) inside the backing cavity. The acoustic field inside the duct and the backing cavity is governed by the Helmholtz equation,

$$
\left(\nabla^{2}-\frac{1}{c_{0}^{2}} \frac{\partial^{2}}{\partial t^{2}}\right) \phi=0
$$

where $c_{0}$ is the speed of sound in air, and $\phi$ is the velocity potential which is related to sound pressure $p$ and acoustic particle velocity $u$ as

$$
p=-\rho_{0} \frac{\partial \phi}{\partial t}, \quad u=\nabla \phi,
$$

with $\rho_{0}$ being the density of air. Under the acoustic excitation, the perforated panel vibrates and the structural vibration is then coupled with the acoustic fields on both sides. When light weight MPP is used, the effect of the structural vibration on the sound absorption characteristics of the MPP absorber can be considerable at the resonance frequencies of the perforated panel. ${ }^{17,18}$ The sound induced vibration of the perforated panel is governed by the dynamic equation,

$$
\left\{\begin{array}{l}
D_{s}(1+j \times \xi) \nabla^{4} w(y, t)+\rho_{s} \frac{\partial^{2} w(y, t)}{\partial t^{2}}=p_{\text {duct }}-p_{\text {cav }}, 0<y<h, \\
w=0, \frac{\partial w}{\partial y}=0, \quad y=0, h, \text { fixed ending }, \\
w=0, \frac{\partial^{2} w}{\partial y^{2}}=0, \quad y=0, h, \text { pinned ending },
\end{array}\right.
$$

where $D_{s}$ and $\xi$ are the flexural rigidity and the loss factor of the perforated panel, $\rho_{s}$ is the MPP surface density, $p_{\text {duct }}$ and $p_{\text {cav }}$ are the sound pressures in the duct and the backing cavity, respectively, $w$ is the normal displacement with the corresponding vibration velocity $u_{s}=j \omega w$ for harmonic timedependence, $h$ is the duct height. The four edges of the MPP (the two ends in the 2D configuration) can be either fixed or simply supported (pinned), depending on the installation scenario chosen.

The acoustic field in the duct is coupled with that in the backing cavity via the structural vibration defined in Eq. (4) as well as the air motion inside the orifices. This vibroacoustic coupling problem is modeled by prescribing an appropriate boundary condition at the interface between the acoustic domains and the perforated panel, as presented below. To simplify the numerical modeling, the discrete air particle velocity over each orifice is averaged across the adjacent unperforated region so that a quasi-continuous particle velocity field $u(y)$ is obtained. By the same token, the acoustic impedance due to the micro-perforations is also averaged and denoted as

$$
Z=Z_{\text {resist }}+j \times Z_{\text {react }},
$$

where $Z_{\text {resist }}$ and $Z_{\text {react }}$ refer to the resistive and reactive parts of the acoustic impedance, respectively. Following Maa's formula, ${ }^{5,7}$ the specific acoustic impedance in Eq. (5) is found as

$$
\begin{aligned}
& Z_{\text {resist }}=\frac{32 \eta t}{\sigma \rho_{0} c_{0} d^{2}}\left[\left(1+\frac{K^{2}}{32}\right)^{1 / 2}+\frac{\sqrt{2}}{32} K \frac{d}{t}\right], \\
& Z_{\text {react }}=\frac{\omega t}{\sigma c_{0}}\left[1+\left(1+\frac{K^{2}}{2}\right)^{-1 / 2}+0.85 \frac{d}{t}\right],
\end{aligned}
$$

where $K=d \sqrt{\omega \rho_{0} / 4 \eta}, d$ is the orifice diameter, $t$ is the panel thickness, $\eta$ is the coefficient of viscosity, and $\sigma$ is the perforation ratio in percentage. Equations (6a) and (6b) have been shown to be accurate enough when the sound pressure level is less than $100 \mathrm{~dB}$ and the orifices are spaced more than one diameter apart. ${ }^{6,7}$ Thus, the averaged velocity field $u(y)$, which combines the normal vibration velocity of the plate $u_{s}$ and the averaged air motion inside the orifice, is found as ${ }^{19}$

$$
u=\zeta_{I} \times u_{s}+\frac{p_{\mathrm{cav}}-p_{\mathrm{duct}}}{\left(\rho_{0} c_{0}\right) Z}
$$

where $\zeta_{I}=1-Z_{\text {react }} \times \sigma / Z$. Applying the continuity condition of normal velocity, the boundary condition at the interfaces between the acoustic domains and the perforated panel is given as,

$$
\frac{\partial \phi}{\partial x}=u
$$

where $\phi$ is the velocity potential defined in the acoustic domains, including both the duct and the backing cavity.

At the left boundary of the duct, the incident sound wave $p_{i}$ is mixed with the unknown reflected sound wave $p_{r}$. An appropriate boundary condition should allow the reflected sound wave to pass through this artificial boundary without reflection. Here, the Dirichlet-to-Neumann boundary condition $^{20}$ is implemented to describe this no-reflection condition. Note that, at the left boundary $\left(x=x_{L}\right)$, the total velocity potential is

$$
\phi=\phi_{i}+\phi_{r}, \quad \text { with } \quad \phi_{i}=-\frac{p_{i}}{j \rho_{0} \omega}, \quad \phi_{r}=-\frac{p_{r}}{j \rho_{0} \omega},
$$

and its normal derivative $\partial \phi / \partial x$ on the boundary is

$$
\frac{\partial \phi}{\partial x}=-j k_{0} \phi_{i}+\frac{\partial \phi_{r}}{\partial x}
$$

The left-going solution for the reflected sound wave $p_{r}$ suggests

C. Wang and L. Huang: Micro-perforated panel absorber array 


$$
\phi(x, y)=\sum_{m=0,1, \ldots} A_{m} \times \Psi_{m}(y) \times e^{j k_{m} x}, \text { at } x \leq x_{L} .
$$

In Eq. (11), $\Psi_{m}(y)$ is the duct acoustics modes of a $2 \mathrm{D}$ channel of height $h$,

$$
\Psi_{m}(y)=\sqrt{2-\delta_{0 m}} \cos (m \pi y / h),
$$

where $\delta_{0 m}$ is the Kronecker $\delta$ and

$$
k_{m}=-j \times \sqrt{(m \pi / h)^{2}-\left(\omega / c_{0}\right)^{2}} .
$$

Owing to the orthogonal property of $\Psi_{m}(y)$, the coefficient $A_{m}$ is evaluated at the left boundary as

$$
A_{m}=\frac{1}{h} \int_{0}^{h}\left(\phi-\phi_{i}\right) \Psi_{m}(y) \mathrm{d} y
$$

Thus, the no-reflection condition at the left boundary is expressed as

$$
\frac{\partial \phi}{\partial x}=-j k_{0} \cdot \phi_{i}+\sum_{m=0}^{M} j k_{m} A_{m} \Psi_{m}(y),
$$

in which $\phi_{i}$ is determined by the given incident pressure $p_{i}$, referring to Eq. (9); the coefficient $A_{m}$ is determined in Eq. (14); $M$ is the highest acoustic mode number used. The walls of the duct and the cavity are assumed to be acoustically rigid, which implies that the particle velocity normal to the walls vanishes,

$$
\frac{\partial \phi}{\partial n}=0
$$

where $n$ denotes the outward normal direction to the wall.

In short, the modeling procedure of the MPP absorber can be summarized as follows. The finite element model contains two acoustic domains (i.e., the duct and backing cavity) and one structural domain. The acoustic problem for velocity potential $\phi$ is specified in Eq. (2) together with boundary conditions of Eqs. (8), (15), and (16). For the structural domain, the bending vibration of the MPP is described by Eq. (4). The effect of the micro-perforations of the MPP is modeled as equivalent acoustic impedance in Eq. (7). The coupling among the two acoustic domains and the structural domains is established in two aspects. First, the structural vibration velocity $u_{s}=j \omega w$ determined by Eq. (4) and the average velocity $u$ determined by Eq. (7) are used to prescribe the boundary condition defined by Eq. (8) in the acoustic domains. Second, the sound pressures $p_{\text {duct }}$ and $p_{\text {cav }}$ in Eqs. (4) and (7) are evaluated in the acoustic domains via the relations in Eq. (3). The governing Eqs. (2) and (4) together with the relevant boundary conditions are solved using COMSOL Multiphysics, which is a commercial finite element software package for solving partial differential equations. With the aid of Eq. (3), the normal incidence absorption coefficient of the MPP absorber can be evaluated either at the left boundary of the duct or at the MPP surface as

$$
\alpha_{N}=\frac{\rho_{0} c_{0} \int_{0}^{h} \operatorname{Re}\left[p^{*}(y) \times u(y)\right] \mathrm{d} y}{\left|p_{i}\right|^{2} h},
$$

where Re means the real part of a complex value and the asterisk denotes the complex conjugate. In the above finite element model, the vibration effect of the MPP is considered in Eq. (4). If the MPP is assumed to be rigid, Eq. (4) is excluded from the numerical model and the corresponding vibration velocity $u_{s}$ in Eq. (7) is then dropped.

As a preliminary validation of the finite element modeling procedure, the sound absorption characteristics of two single MPP absorbers are simulated and compared with the analytical solutions available in literatures. The first numerical example consists of an MPP fitted in front of a solid wall with a constant air gap of $D=100 \mathrm{~mm}$. The perforated panel is assumed to be rigid so that the vibration effect is not considered. Other properties of the MPP are $d=t=0.5 \mathrm{~mm}$ and $\sigma=1 \%$, where $d$ is the orifice diameter, $t$ is the panel thickness, and $\sigma$ is the perforation ratio. For such a simple configuration, the normal incidence absorption coefficients can be calculated analytically following the equivalent electrical circuit method in Maa, ${ }^{5,6}$ as shown in Fig. 3(a). In the finite element simulation, the constant air gap between the MPP and the solid wall is modeled as a rectangular cavity of the size $200 \mathrm{~mm}$ (width) $\times 100 \mathrm{~mm}$ (depth), and the infinite perforated panel is reduced to a beam of finite length $L=200 \mathrm{~mm}$. The computational domains are discretized with triangular quadratic-Lagrange elements. The maximum element size is set to be $0.01 \mathrm{~m}$ and a total of 5096 elements are defined. The same meshing scheme applies to the following 2D simulations in Sec. III. The predicted absorption coefficients by the finite element simulation are shown in Fig. 3(a) in open circles. In the second numerical example, the normal incidence absorption coefficients of a finite flexible MPP backed by a rectangular air cavity are simulated and compared with the results obtained with the modal analysis method. ${ }^{17}$ The dimension of the air cavity is $200 \mathrm{~mm}$ (length) $\times 200 \mathrm{~mm}$ (width) $\times 150 \mathrm{~mm}$ (depth). The first structural resonance of the perforated panel is set to be $700 \mathrm{~Hz}$, with the loss factor $\xi=0.04$ and the surface density $\rho_{s}=1 \mathrm{~kg} /$ $\mathrm{m}^{2}$. The properties of the MPP are $d=0.4 \mathrm{~mm}, t=0.3 \mathrm{~mm}$, $\sigma=1 \%$. The four edges of the perforated panel are simply supported. To accurately model the structural vibration effect, a full three-dimensional (3D) finite element model is established following the same procedure as in the $2 \mathrm{D}$ configuration. The $3 \mathrm{D}$ acoustic domain is meshed with a total of 4836 prism quadratic-Lagrange elements (maximum element size: 0.03 $\mathrm{m})$. The simply supported flexible panel, which corresponds to Eq. (4), is meshed with a total of 744 triangular quadraticLagrange elements. The predicted sound absorption coefficients by the two methods are compared in Fig. 3(b). In both cases, the finite element simulations are in good agreement with the analytical solutions. For a complicated configuration like the MPP absorber array with modulated cavity depth, the analytical methods are no longer suitable for the prediction. For verification purpose, experimental studies are carried out in Sec. IV and the measured absorption coefficients are compared with the finite element simulations. 

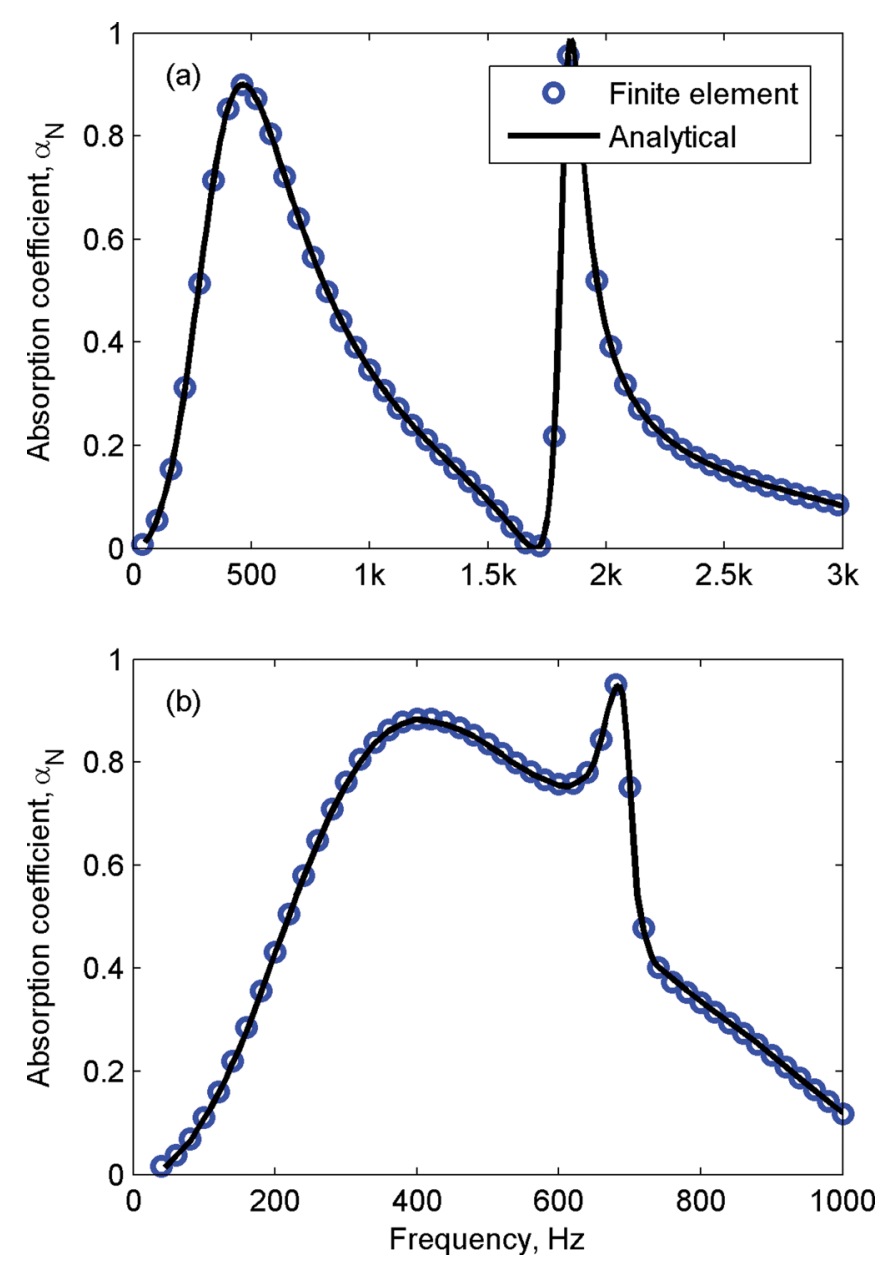

FIG. 3. (Color online) Comparison of the predicted normal incidence absorption coefficients by the finite element model and the analytical solutions. (a) Rigid MPP. The 2D rectangular cavity is of the size $200 \mathrm{~mm}$ (width) $\times 100 \mathrm{~mm}$ (depth). The properties of the MPP are, $d=t=0.5 \mathrm{~mm}$, perforation ratio $\sigma=1 \%$. (b) Flexible MPP. The 3D cavity is of the size 200 $\mathrm{mm}$ (length) $\times 200 \mathrm{~mm}$ (width) $\times 150$ (depth). The first structural resonance of the MPP is $700 \mathrm{~Hz}$. The MPP parameters are, $d=0.4 \mathrm{~mm}, t=0.3 \mathrm{~mm}$, perforation ratio $\sigma=1 \%$. The surface density of the MPP is $\rho_{S}=1 \mathrm{~kg} / \mathrm{m}^{2}$.

\section{NUMERICAL RESULTS}

The acoustic behaviors of the MPP absorber array are investigated numerically using the finite element procedure presented above. As shown in Fig. 1(b), the MPP absorber array consists of three different cavities partitioned by rigid walls. Studies show that a combination of more cavities may achieve better absorption performance. But the three-cavity configuration is sufficient to demonstrate the distinct acoustic properties of the MPP absorber array while keeping the structure relatively simple and easy to analyze. Likewise, the structural vibration effect of the perforated panel is excluded in the following simulations. The default geometry of the MPP absorber array is set as follows,

$$
\begin{aligned}
& D_{1}=50 \mathrm{~mm}, \quad D_{2}=100 \mathrm{~mm}, D_{3}=25 \mathrm{~mm}, \\
& W_{1,2,3}=30 \mathrm{~mm} .
\end{aligned}
$$

Note that the choice of the above geometrical parameters is a little bit arbitrary. No effort is made to optimize the absorption performance. Also note that the widths of the three cavities,
TABLE I. Properties of the MPPs used in the numerical investigations.

\begin{tabular}{lccc}
\hline \hline Orifice diameter & Thickness & Perforation ratio & Acoustic resistance \\
\hline $0.3 \mathrm{~mm}$ (small) & $0.5 \mathrm{~mm}$ & $1 \%$ & sufficient \\
$0.5 \mathrm{~mm}$ (moderate) & $0.5 \mathrm{~mm}$ & $1 \%$ & moderate \\
$0.8 \mathrm{~mm}$ (large) & $0.5 \mathrm{~mm}$ & $1 \%$ & insufficient \\
\hline \hline
\end{tabular}

$W_{1}, W_{2}$, and $W_{3}$, are not necessarily equal to each other. In real design situations, a parametric study can be performed to determine the optimal geometrical configuration.

\section{A. Sound absorption coefficients under normal incidence}

Three types of MPPs with different orifice diameters are investigated, as listed in Table I. The relative acoustic resistance of the MPPs is plotted in Fig. 4 for reference. For the single MPP absorber, it is well known that the good absorption performance requires that the acoustic resistance $Z_{\text {resist }}$ of the MPP match that of air $Z_{\text {air }}=1$. A small orifice diameter $d=0.3 \mathrm{~mm}$ can provide sufficient acoustic resistance for good sound absorption. As can be observed in Fig. 4, $Z_{\text {resist }}$ varies nearly proportionally to the reciprocal of the orifice diameter $d$. When the orifice diameter increases to $d=0.8$ $\mathrm{mm}, Z_{\text {resist }}$ becomes far below the optimal value $\left(Z_{\text {air }}=1\right)$, especially at the low frequencies. Based on the impedance matching conditions for the single MPP absorber, the acoustic resistance is denoted as "sufficient," "moderate," and "insufficient," respectively. Certainly, with the orifice diameter $d=0.8 \mathrm{~mm}$ given, the acoustic resistance of the MPP can be improved to the sufficient condition $Z_{\text {resist }} \approx 1$ with a smaller perforation ratio, for example, $\sigma=0.3 \%$, but the resulting absorption bandwidth shrinks dramatically. A comprehensive analysis of the choice of perforation parameters for broadband absorption can be found in Ref. 8 .

Figure 5 compares the normal incidence absorption coefficients between the MPP absorber array and the corresponding single MPP absorbers under different acoustic resistance conditions. The single MPP absorber is simulated

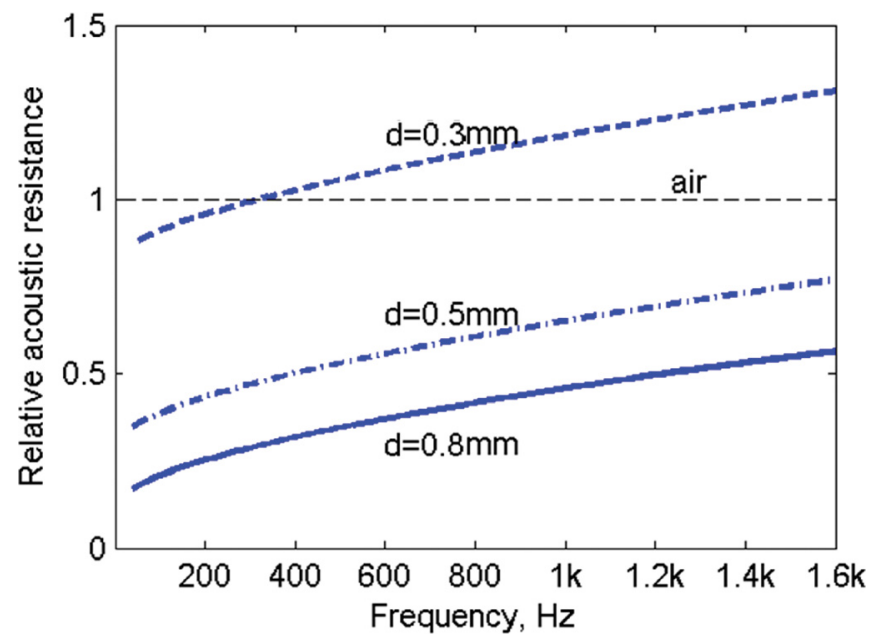

FIG. 4. (Color online) The acoustic resistance (relative to air) of MPPs with different orifice diameters. Other parameters: thickness $t=0.5 \mathrm{~mm}$, perforation ratio $\sigma=1 \%$

C. Wang and L. Huang: Micro-perforated panel absorber array 

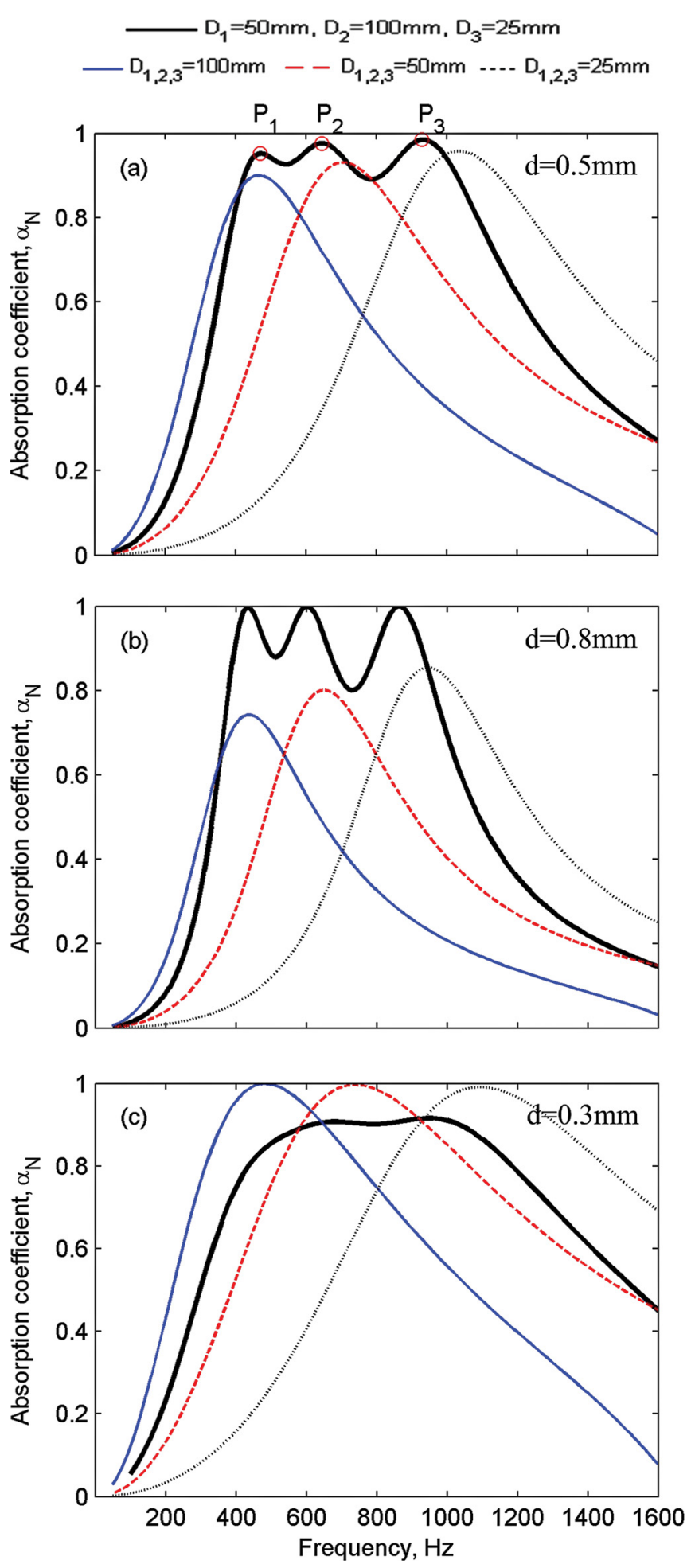

FIG. 5. (Color online) Comparison of the normal incidence absorption coefficients between MPP absorber array $\left(D_{1}=50 \mathrm{~mm}, D_{2}=100 \mathrm{~mm}\right.$, $\left.D_{3}=25 \mathrm{~mm}\right)$ and single MPP absorbers $\left(D_{1,2,3}=25 \mathrm{~mm}, 50 \mathrm{~mm}\right.$, and $100 \mathrm{~mm}$, respectively). (a) Orifice diameter $d=0.5 \mathrm{~mm}$; (b) Orifice diameter $d=0.8 \mathrm{~mm}$; (c) Orifice diameter $d=0.3 \mathrm{~mm}$. Other parameters: cavity width $W=30 \mathrm{~mm}$, panel thickness $t=0.5 \mathrm{~mm}$, perforation ratio $\sigma=1 \%$.

by setting the depths of the three cavities equal to each other, that is, $D_{1,2,3}=D$. Figure 5(a) shows the results for the MPP with moderate acoustic resistance $(d=0.5 \mathrm{~mm})$. The thick solid curve is for the MPP absorber array with $D_{1}=50 \mathrm{~mm}, D_{2}=100 \mathrm{~mm}$, and $D_{3}=25 \mathrm{~mm}$. Three spectral peaks are observed at $P_{1,2,3}=470 \mathrm{~Hz}, 650 \mathrm{~Hz}$ and $930 \mathrm{~Hz}$ with $\alpha_{N}>0.95$. Between the three peaks, the sound absorption is still maintained at a reasonably high level, say, $\alpha_{N}>0.9$. The results for the single MPP absorber with $D_{1,2,3}=100 \mathrm{~mm}, 50 \mathrm{~mm}$, and $25 \mathrm{~mm}$ are plotted in thin solid, dashed, and dotted curves. The corresponding resonance frequencies are $465 \mathrm{~Hz}, 700 \mathrm{~Hz}$, and $1030 \mathrm{~Hz}$. Compared with the single MPP absorber, two features of the MPP absorber array can be identified. First, the local resonances of the MPP absorber array resemble those of the individual MPP absorbers, but noticeable frequency shifts can be observed. The frequency shift can be attributed to the inter-resonator interaction, and it should be taken into account at the design stage. One more important feature of the MPP absorber array is the greatly enhanced sound absorption associated with the local resonances, which is usually neglected in previous investigations. As shown in Fig. 5(a), although only one portion of the MPP is in resonance at one particular spectral peak, the overall sound absorption coefficients can be higher than those of the corresponding single MPP absorbers, in which the whole MPP is in resonance. The performance enhancement is found to be closely related to the value of the relative acoustic resistance of the MPP, as shown below.

Figure 5(b) shows the results for the MPP with relatively large orifice diameter $d=0.8 \mathrm{~mm}$. Referring to Fig. 4 , the large perforations cannot provide sufficient acoustic resistance for the single MPP absorbers. As a result, the corresponding absorption coefficients drop approximately by $15 \%-20 \%$ as the orifice diameter increases from $d=0.5$ $\mathrm{mm}$ to $d=0.8 \mathrm{~mm}$. In contrast, for the MPP absorber array, the absorption coefficients are still maintained at a high level on the whole. Moreover, almost complete sound absorption is achieved at the three resonances, which suggests that the required optimal acoustic resistance of the MPP absorber array is much lower than that of the single MPP absorbers. As in this case, the relative acoustic resistance of the MPP at the three resonances is $Z_{\text {resist }}=0.32$, 0.37 , and 0.43 , respectively. Considering the excess cost associated with the manufacturing of minute orifices in the MPP, the lower requirement of acoustic resistance in the MPP absorber array constitutes a beneficial property as it can be reached much more easily with relatively larger perforations.

Figure 5(c) shows the results for the MPP with small orifice diameter $d=0.3 \mathrm{~mm}$. For the single MPP absorbers, almost complete sound absorption is achieved at the resonance frequencies due to the good impedance matching. However, the MPP absorber array seems to be over-damped. As shown by the solid curve in Fig. 5(c), for the MPP absorber array, the resonance peaks in the absorption curve are smoothed out; and the peak absorption coefficient drops to around $\alpha_{N}=0.9$. This again suggests the different impedance matching conditions between the single absorber and the absorber array because of the possible different absorption mechanisms. Aiming for the enhanced absorption performance of the MPP absorber array, lower acoustic impedance than the single MPP absorber is preferred. Reasons for this apparent difference are discussed in Sec. III B. 

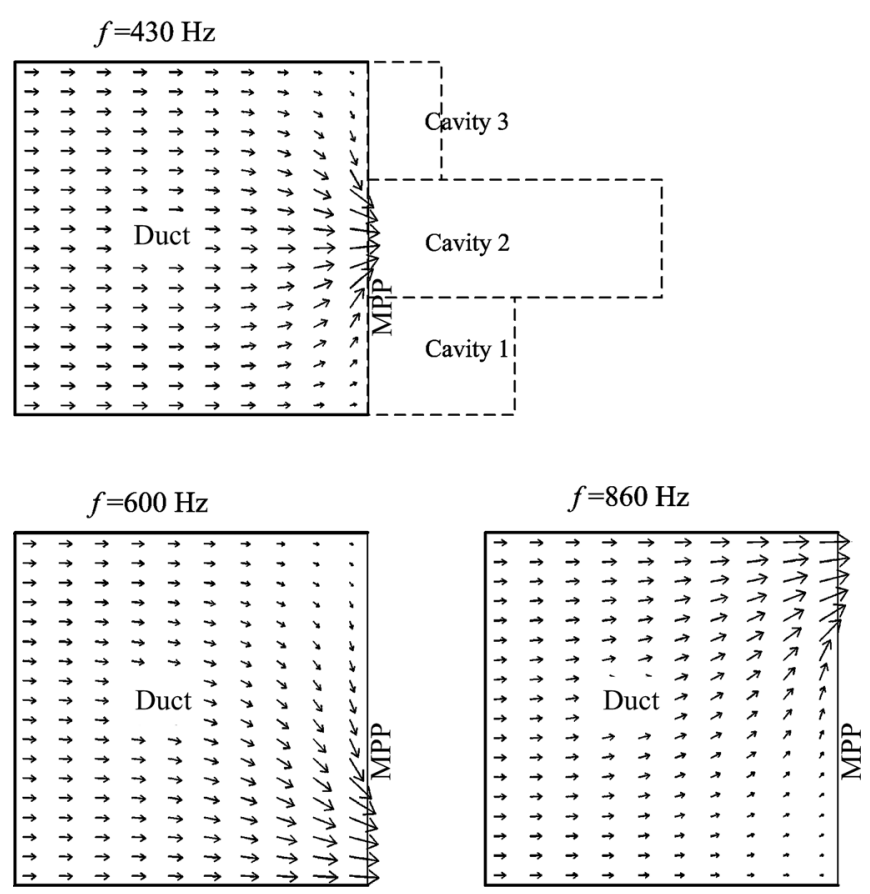

FIG. 6. The acoustic intensity in the duct at the three resonance frequencies of the MPP absorber array. The backing cavities are shown in dashed lines in the subfigure for $f=430 \mathrm{~Hz}$ for reference purpose. The geometrical configurations and the MPP parameters are the same as in Fig. 5(b).

\section{B. Local resonances and inter-resonator interaction}

The unequal cavity depths create different reactance matching conditions for each individual MPP absorber, which in turn causes strong local resonance absorption and out-of-phase air motion across the MPP surface. With the aid of the finite element model, the local resonances and the corresponding inter-resonator interaction are examined numerically, and their effects on the acoustic properties of the MPP absorber array are discussed accordingly.

Figure 6 depicts the acoustic intensity in the duct at $f=430,600$, and $860 \mathrm{~Hz}$, corresponding to the three spectral peaks in Fig. 5(b), respectively. The backing cavities are shown in dashed lines in the first subfigure for reference. At $f=430 \mathrm{~Hz}$, cavity 2 is excited to resonance as the inertia effect of the air mass in the orifice is balanced by the stiffness effect of the backing cavity. It can be seen that most part of the acoustic energy is "attracted" toward this cavity and dissipated by the MPP covering it. At $f=600$ and $860 \mathrm{~Hz}$, cavity 1 and cavity 3 get to resonance, respectively. Similar observations can be made at these two resonance frequencies. The contributions of the three component MPP absorbers toward the total sound absorption are assessed quantitatively and the results are shown in Fig. 7. The sound absorption coefficient by the $i$ th $(i=1,2,3)$ component MPP absorber is evaluated as

$$
\alpha_{N}^{i}=\frac{E_{\text {absorbed }}^{i}}{E_{\text {incident }}}
$$

where $E_{\text {incident }}$ denotes the total incident acoustic energy, $E_{\text {absorbed }}^{i}$ denotes the acoustic energy dissipated by the designated component MPP absorber. The absorption coefficients for the single MPP absorber are shown in Fig. 5(b). One ob-

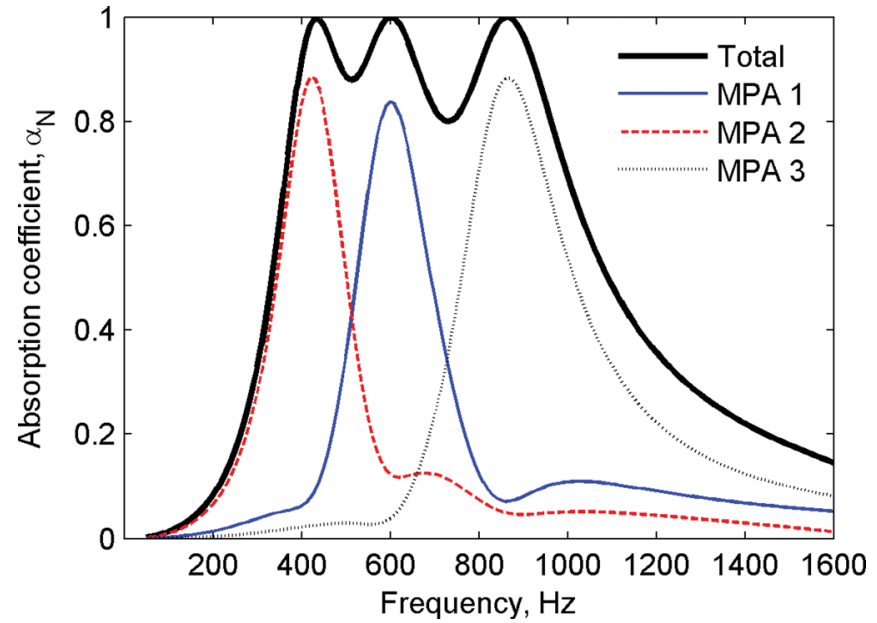

FIG. 7. (Color online) Contribution of the three component MPP absorbers toward the total sound absorption coefficient. MPA 1-3 denotes the MPP patch covering cavity $1-3$, respectively.

servation is that the resonating component MPP absorber can dissipate more sound energy than the corresponding single MPP absorber, although only one third of the total MPP is in resonance. In fact, by removing the acoustic energy absorbed due to the non-resonating absorbers from $E_{\text {incident }}$, the spectral peaks for $\alpha_{N}^{i}$ approach to unity, which implies complete sound absorption occurs within the resonating component MPP absorber.

The inter-resonator interaction between the resonating and non-resonating component MPP absorbers is examined at the second resonance frequency of $f=600 \mathrm{~Hz}$. Figure 8 shows the distribution of the instantaneous particle velocity at the phase angle $\omega t=0, \pi / 2, \pi$, and $3 \pi / 2$. The locations of the cavities are the same as in Fig. 6. In the far field of the MPP, a flat wavefront is observed as it would be when the MPP is
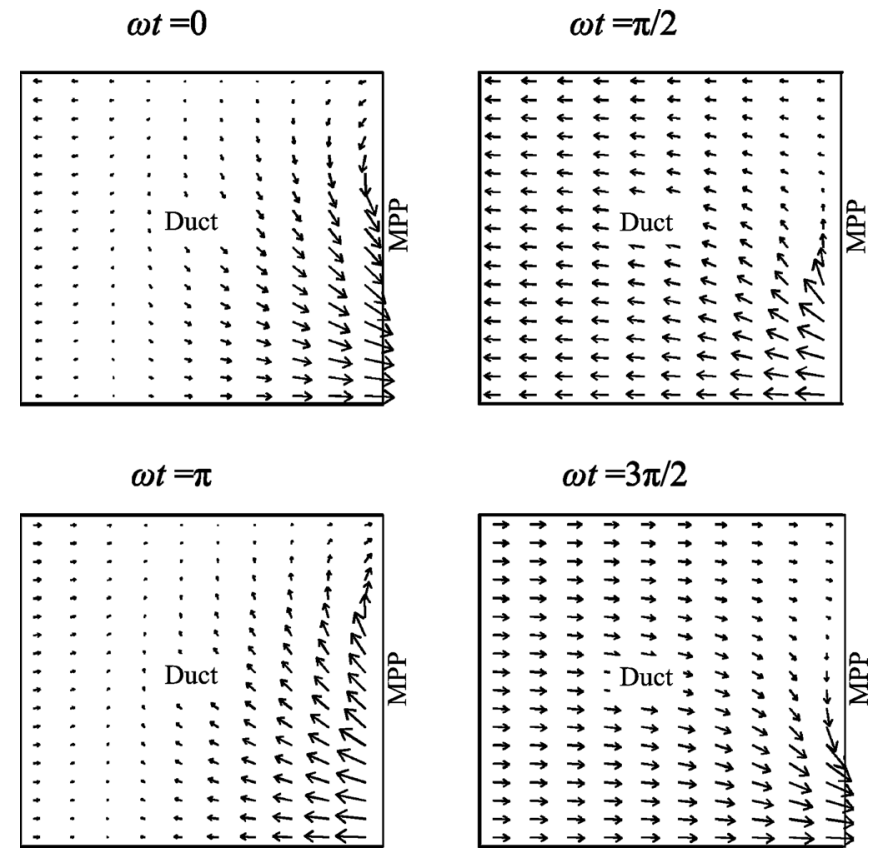

$\omega t=3 \pi / 2$

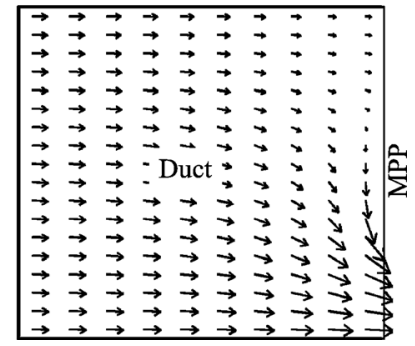

FIG. 8. Spatial distribution of the instantaneous particle velocity at $f=600$ $\mathrm{Hz}$ in one period. The design parameters and the locations of the backing cavities are the same as in Fig. 6. 

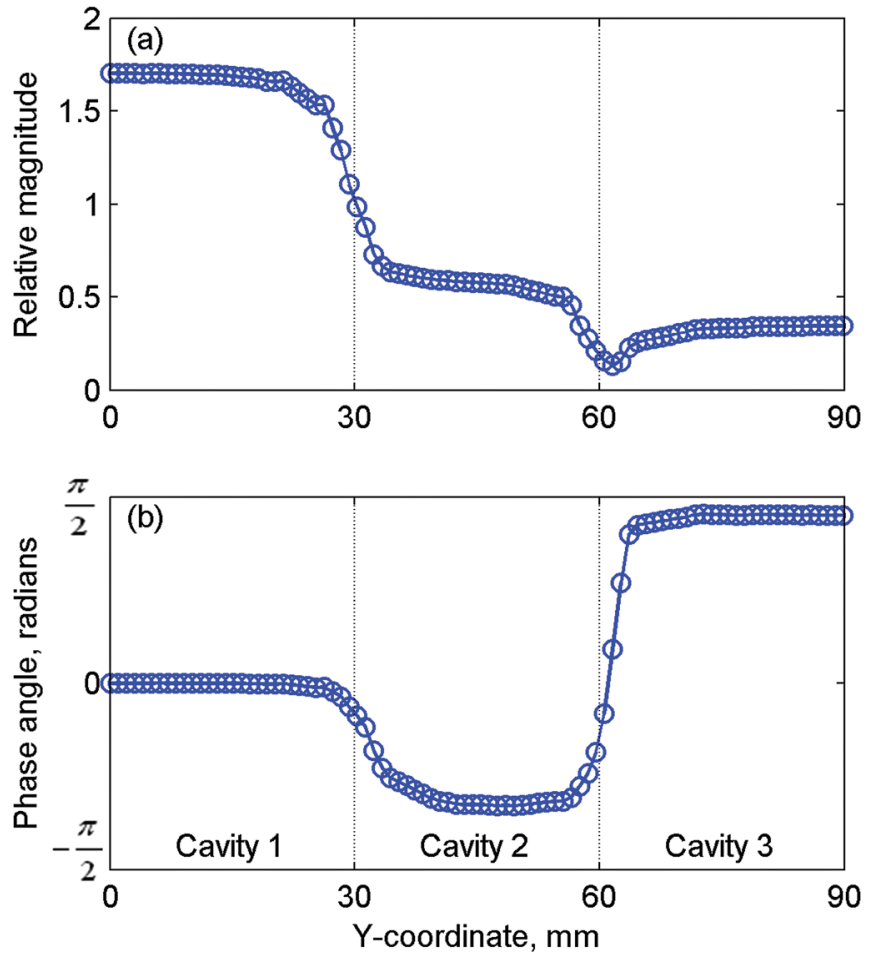

FIG. 9. (Color online) The normal particle velocity over the MPP surface at $f=600 \mathrm{~Hz}$. (a) Magnitude, normalized to the particle velocity in the corresponding single MPP absorber at its resonance frequency $(f=640 \mathrm{~Hz})$. (b) Phase angle.

backed by a constant air gap. In the near field of the MPP, however, the wavefront is distorted greatly because of different reactance matching conditions across the MPP surface. Obvious acoustic wave propagation between cavity 1 and cavity 3 can be observed at the given frequency. Note that the air motion around the two non-resonating cavities is out of phase with that around the resonating region (cavity 1). This phase difference is caused by the different distances that the acoustic wave travels within each individual cavity. Figure 9 shows the normal particle velocity over the MPP surface. The magnitude is normalized to the particle velocity in the corresponding single MPP absorber at its resonance frequency $(f=640$ $\mathrm{Hz}$ ). Due to the strong local resonance, the sound flux is attracted to cavity 1 , and the air motion there is greatly intensified. As shown in Fig. 9, the magnitude of the normal particle velocity over cavity 1 is about 1.7 times as large as that in the single MPP absorber. Also, there are flows in and out the micro-perforations over the non-resonating cavities, but the magnitude is only in the fraction of the resonating one.

The sound absorption mechanism in the parallel arrangement of multiple MPP absorber array, or "the parallel absorption mechanism," is now analyzed. As shown in Figs. 6 and 7, the strong local resonance accounts for most of the sound absorption. Because of the different reactance matching conditions, the component MPP absorbers get to resonance at different frequencies, and most of the acoustic energy is attracted to the resonating MPP absorber and dissipated there. The supplementary sound absorption by the non-resonating MPP absorbers is trivial. Thus, as far as the sound absorption is concerned, it is reasonable to assume that the non-resonating component absorbers do not work. In other words, the MPP patches covering the non-resonating absorbers are regarded as non-perforating hard wall. Under this assumption, the effective perforation ratio for the whole MPP is reduced to $1 / 3$ of the original one. Accordingly, the effective acoustic resistance is increased to three times its nominal value, referring to Eq. 6(a). In the light of this analysis, for the MPP absorber array, the effective acoustic resistance of the MPP with $d=0.8 \mathrm{~mm}$ will be $Z_{\text {resist }}^{\prime}=0.96$, 1.11 , and 1.29 at the three resonance frequencies, which just satisfies the well-known impedance matching conditions for good sound absorption performance. For the same reason, if the MPP is designed for optimal impedance matching in the single MPP absorbers, the acoustic resistance may be too high for the MPP absorber array to meet the impedance matching condition, as is the case shown in Fig. 5(c). Thus, the apparent difference in the acoustic resistance matching conditions between the single MPP absorber and the MPP absorber array is explained.

On the other hand, the out-of-phase air motion between different component MPP absorbers changes the effective acoustic reactance of the MPP patches covering the resonating absorber, which accounts for the frequency shift of the resonances in the MPP absorber array. This effect is analyzed qualitatively below. Take the third spectral peak in Fig. 5, for example, since its frequency shift is the most pronounced. This spectral peak corresponds to the local resonance of cavity 3 with the shortest depth $D_{3}=25 \mathrm{~mm}$. In the single MPP absorber, it is known that resonance occurs when the reactance of the MPP is balanced by the stiffness effect of the backing cavity, that is,

$$
Z_{\text {react }}-\cot \left(\omega D_{3} / c_{0}\right)=0
$$

In the MPP absorber array, when the above reactance matching condition is reached, the residual reactance at the other two cavities is always positive because of the longer cavity depths. That is,

$$
Z_{\text {react }}-\cot \left(\omega D_{1} / c_{0}\right)>0, Z_{\text {react }}-\cot \left(\omega D_{2} / c_{0}\right)>0
$$

The positive reactance at the neighboring cavities imposes an added-mass effect on the MPP patch covering cavity 3 . Therefore, the effective acoustic reactance of the MPP is larger than the nominal value $Z_{\text {react }}$ when the shortest cavity is in resonance. As a result, the resonance frequency shifts to lower frequency.

The effect of the finite geometry of the MPP absorber array on the parallel absorption mechanism is investigated by varying the cavity width and their space arrangements. Figure 10 shows the variation of the predicted absorption coefficients with the cavity width. The default cavity depths are used. The parameters of the MPP are specified in the last row of Table $\mathrm{I}(d=0.8 \mathrm{~mm})$. As the cavity width increases from $W=20 \mathrm{~mm}$ to $W=50 \mathrm{~mm}$, the second and the third peaks shift to lower frequencies, and the off resonance absorption performance decreases. As the cavity width further increases to $W=80 \mathrm{~mm}$, the sound absorption level at the first two peaks remains almost unchanged, but the 


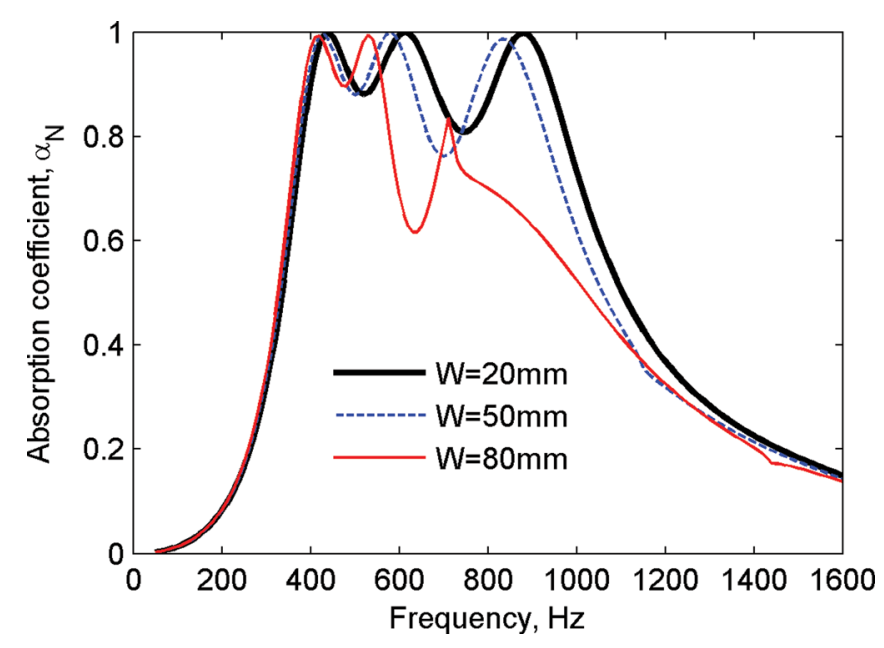

FIG. 10. (Color online) Variation of the normal incidence absorption coefficients with the cavity width. The cavity depths are $D_{1}=50 \mathrm{~mm}$; $D_{2}=100 \mathrm{~mm} ; D_{3}=25 \mathrm{~mm}$. The MPP parameters are: $d=0.8 \mathrm{~mm}$, $t=0.5 \mathrm{~mm}, \sigma=1 \%$.

absorption performance around the third peak drops dramatically. In the frequency range from $700 \mathrm{~Hz}$ to $1000 \mathrm{~Hz}$, the absorption coefficient of the absorber array is approximately the average of the component MPP absorbers, which implies that the component MPP absorbers work almost separately. Note that the center-to-center distance between cavity 1 and cavity 3 is $2 \times W=160 \mathrm{~mm}$, which approximately equals to a quarter wavelength of the acoustic wave at $f=540 \mathrm{~Hz}$. This frequency is roughly regarded as the critical frequency, beyond which the above-mentioned parallel absorption mechanism cannot sustain, as can be observed in Fig. 10. From this investigation, one conclusion is that the parallel absorption mechanism works only when the distance between the component MPP absorbers is less than a quarter-wavelength of the acoustic wave.

Figure 11 shows the effect of space arrangement on the sound absorption performance. In the finite element model, two arrangement sequences of the cavities are considered:

$$
\begin{aligned}
& D_{1}=50 \mathrm{~mm}, \quad D_{2}=100 \mathrm{~mm}, \\
& D_{3}=25 \mathrm{~mm}, \quad W_{1,2,3}=50 \mathrm{~mm}, \\
& D_{1}=50 \mathrm{~mm}, \quad D_{2}=25 \mathrm{~mm}, \\
& D_{3}=100 \mathrm{~mm}, \quad W_{1,2,3}=50 \mathrm{~mm} .
\end{aligned}
$$

The corresponding results are indicated as "FEM, Sequence 1" and "FEM, Sequence 2" in Fig. 11. For different arrangement sequences, the absorption curves are similar on the whole with slightly different resonance frequencies. The major difference lies in the variations of the off resonance absorption performance. The variation can be very pronounced when the cavity width is large relative to the acoustic wavelength. For comparison, the results by the electrical circuit model are calculated and shown in solid curve. The electrical circuit model represents the ideal parallel absorption mechanism with the cavity width reduces to infinitesimal. In general, the off resonance performance predicted by the electrical circuit mode is better than the finite element simulations except the second trough in "FEM, Sequence 2." It indicates that the overall effect of the finite geometry is counter-productive for the sound absorption at the off resonance frequencies.

\section{EXPERIMENTAL STUDIES}

Experimental studies are carried out to validate the finite element model as well as the numerical findings about the MPP absorber array. The prototype consists of three cavities with depths $D_{1,2,3}=24 \mathrm{~mm}, 48 \mathrm{~mm}$, and $98 \mathrm{~mm}$, respectively. The three cavities are separated with two pieces of steel plate of thickness $1 \mathrm{~mm}$. The cavity walls are made of acrylic of thickness $15 \mathrm{~mm}$. The properties of the MPP are

$$
d=0.64 \mathrm{~mm}, \quad t=0.5 \mathrm{~mm}, \quad \sigma=1.6 \% .
$$

The MPP is made of stainless steel. The normal incidence absorption coefficients of the prototype MPP absorber array are measured experimentally and compared with the numerical predictions. The test rig is designed based on the two-microphone transfer-function method according to

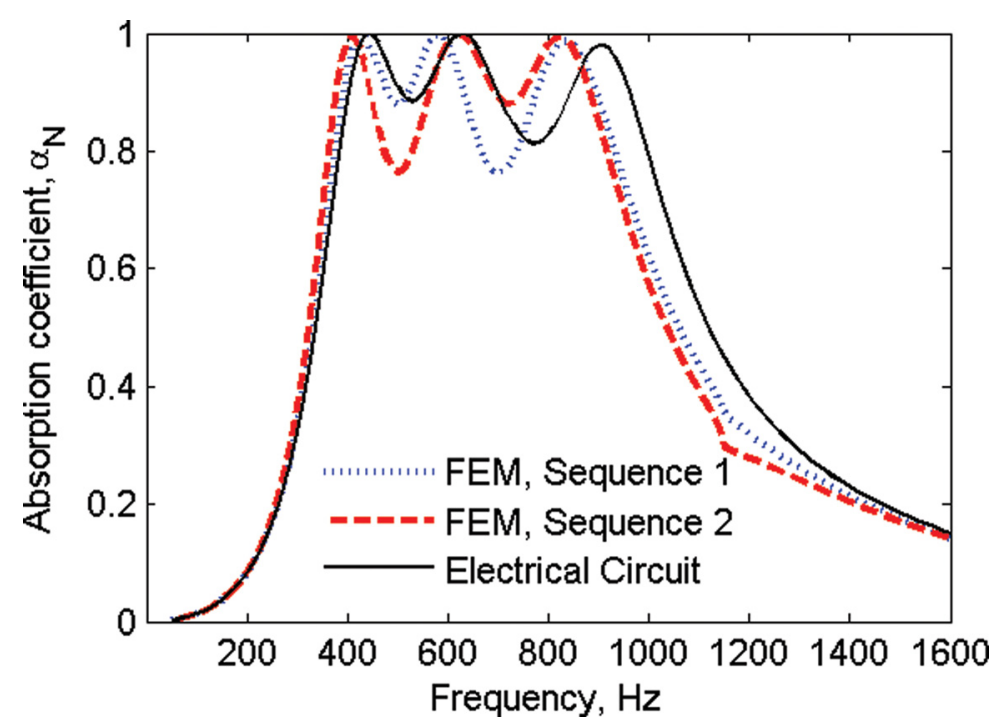

FIG. 11. (Color online) Variation of the absorption coefficients with the arrangement sequences of the cavities in the MPP absorber array. Sequence 1: $D_{1}=50 \mathrm{~mm} ; D_{2}=25 \mathrm{~mm}$; $D_{3}=100 \mathrm{~mm}$. Sequence 2: $D_{1}=25 \mathrm{~mm} ; D_{2}=50 \mathrm{~mm}$; $D_{3}=100 \mathrm{~mm}$. The cavity width is $W_{1,2,3}=50 \mathrm{~mm}$. Results by the electrical circuit model are shown for reference. 

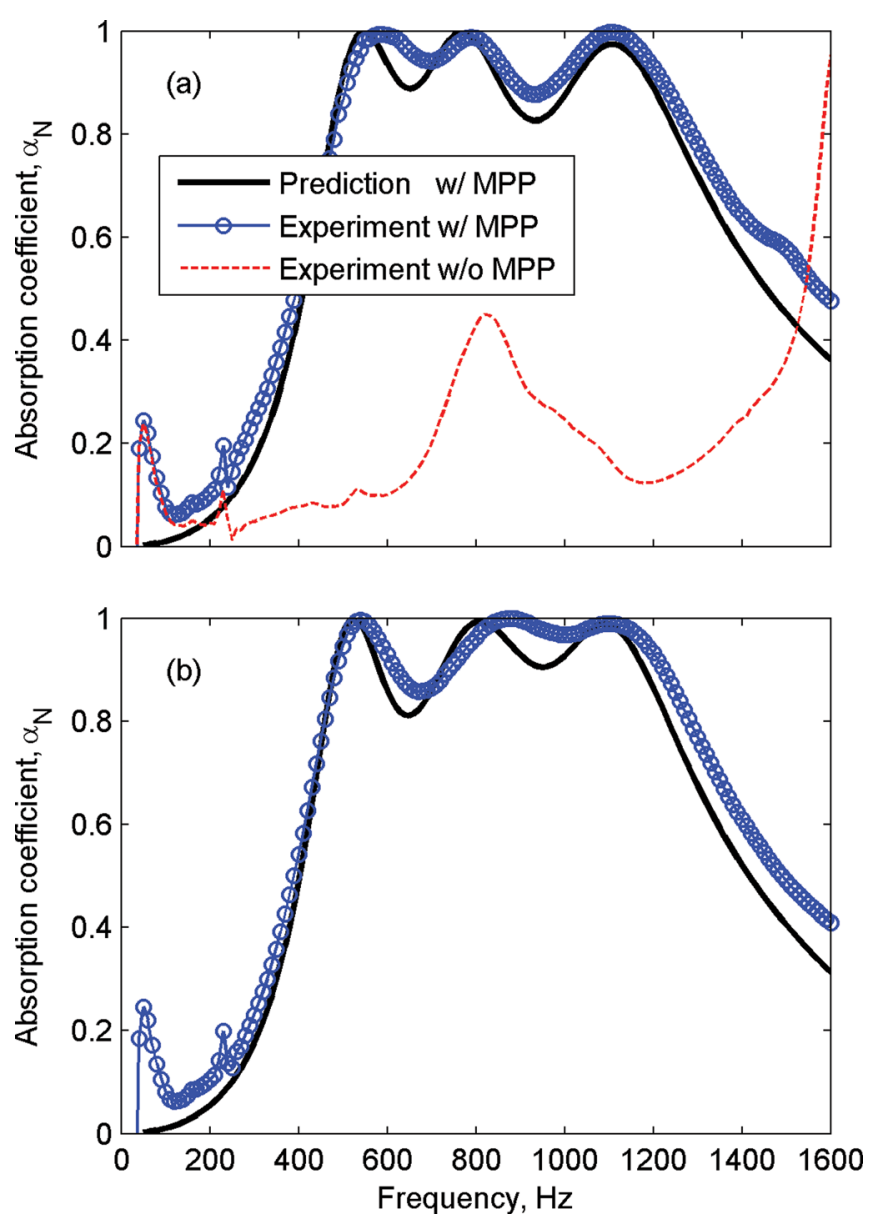

FIG. 12. (Color online) The normal incidence absorption coefficients of the prototype MPP absorber array. (a) $D_{1}=24 \mathrm{~mm}, D_{2}=98 \mathrm{~mm}, D_{3}=48 \mathrm{~mm}$. The dashed curve shows the measured absorption coefficients when the MPP is removed. (b) $D_{1}=24 \mathrm{~mm} ; D_{2}=48 \mathrm{~mm} ; D_{3}=98 \mathrm{~mm}$.

ISO-10534-2 (1988). ${ }^{16}$ The straight rectangular duct is also made of acrylic of thickness $15 \mathrm{~mm}$ so that the duct walls can be regarded as acoustically rigid. The cross section of the rectangular duct is of $100 \mathrm{~mm} \times 100 \mathrm{~mm}$. So, the first cut-on frequency in the rectangular duct is around 1700 $\mathrm{Hz}$.

Figure 12 compares the predicted and measured normal incidence absorption coefficients of the prototype MPP absorber array. Two cavity sequences are considered:

$$
\begin{aligned}
& D_{1}=24 \mathrm{~mm}, \quad D_{2}=98 \mathrm{~mm}, \quad D_{3}=48 \mathrm{~mm}, \\
& D_{1}=24 \mathrm{~mm}, \quad D_{2}=48 \mathrm{~mm}, \quad D_{3}=98 \mathrm{~mm} .
\end{aligned}
$$

The results are shown in Figs. 12(a) and 12(b), respectively. The solid curves are for the numerical predictions. Within the frequency range investigated, three peaks can be observed in both cases. As already mentioned in Sec. III, the arrangement sequences influence the absorption levels between the adjacent peaks because of the finite geometry effect. The solid curves with open circles show the measured absorption coefficients. In both Figs. 12(a) and 12(b), the experimental results are in good agreement with the predictions in terms of both the peak frequencies and the overall sound absorption levels. The unexpected peaks at $f=50 \mathrm{~Hz}$ and $230 \mathrm{~Hz}$ are caused by the low frequency vibration of the test rig itself. The effect of structural vibration of the perforated panel is not measured as it occurs far beyond the frequency range tested.

The sound absorption performance is also tested with the MPP absent from the prototype MPP absorber array. In this case, the main sound absorption mechanisms are the viscous and thermal losses inside the cavity as well as the structural vibration of the partitioning plates. The measured results are shown in Fig. 12(a) in dashed line. When the MPP is removed, the overall sound absorption level is rather low $\left(\alpha_{N} \leq 0.1\right)$ except around the resonance peaks. This test reveals that the dissipation by the MPP is the dominant sound absorption mechanism in the MPP absorber array, although other mechanisms may exist in the device. In fact, most of the incident acoustic energy will be either dissipated or reflected by the MPP in the absorber array, and only a small fraction of it has the chance of entering the cavity and being dissipated by these extra absorption mechanisms. Note that the device without MPP results in a sharp peak with high absorption rate around $f=1600 \mathrm{~Hz}$, but the absorption curve for the MPP absorber array is rather smooth around that frequency and the corresponding absorption coefficient is low. Thus, as far as the design of the MPP absorber array is concerned, it is appropriate to exclude these extra absorption mechanisms inside the cavity.

\section{CONCLUDING REMARKS}

The acoustic properties of a parallel arrangement of multiple MPP absorbers with different cavity depths are investigated. A finite element procedure is used to simulate the acoustic behaviors of the MPP absorber array under normal incidence. Experimental studies are also carried out to verify the simulation model as well as the numerical findings. The following conclusions are made.

(1) Strong local resonance occurs within the MPP absorber array due to the different reactance matching conditions for the component MPP absorbers. Most of the acoustic energy is attracted toward and absorbed by the resonating MPP absorber. The supplementary sound absorption by the non-resonating MPP absorbers is trivial for the resonance performance. The out-of-phase air motion changes the effective acoustic reactance of the MPP patch covering the resonating cavity, which shifts the resonance frequency. All the above three aspects, that is, the strong local resonance absorption, the supplementary absorption by the non-resonating absorbers and the change of environmental impedance conditions, constitute the parallel absorption mechanism.

(2) Compared with the single MPP absorber, the MPP absorber array requires lower acoustic resistance for good sound absorption performance. In the MPP absorber array, the local resonance absorption reduces the effective perforation ratio of the MPP, which causes the effective acoustic resistance of the MPP in resonance increase accordingly. If this increase in acoustic resistance is taken into account, the acoustic resistance matching conditions for the MPP absorber array and the single MPP absorber are consistent with each other. 
(3) The parallel absorption mechanism is affected by the finite geometry of the MPP absorber array. The absorption performance of the MPP absorber array varies with the widths and spatial arrangement of the backing cavities, especially at the off resonance frequencies. Though marginal performance improvement may occur at certain off resonance frequency, the overall effect of the finite geometry is counter-productive for good sound absorption. Furthermore, the parallel absorption mechanism works only when the distance between the component MPP absorbers is less than a quarter-wavelength of the acoustic wave. Otherwise, the component MPP absorbers work separately, and the overall sound absorption coefficient of the MPP absorber array is approximately the average of the individual MPP absorbers.

(4) The absorption coefficients of a prototype MPP absorber array are tested experimentally using a standing wave tube and compared with the finite element simulations. The experimental results are in good agreement with the numerical predictions. Experimental test also shows that other absorption mechanisms may exist in the MPP absorber array, but the dissipation by the MPP is dominant.

\section{ACKNOWLEDGMENTS}

The research reported here is supported by a grant from the Research Grants Council of the Hong Kong SAR (HKU 7126/08E). The first author thanks the Faculty of Engineering (HKU) for the postdoctoral fellowship.

${ }^{1}$ H. V. Fuchs and X. Zha, "Acrylic-glass sound absorbers in the plenum of the Deutscher Bundestag,” Appl. Acoust. 51, 211-217 (1997).

${ }^{2}$ H. V. Fuchs and X. Zha, "Micro-perforated structures as sound absorbersA review and outlook," Acta. Acust. Acust. 92, 139-146 (2006).

${ }^{3}$ F. Asdrubali and G. Pispola, "Properties of transparent sound absorbing panels for use in noise barriers," J. Acoust. Soc. Am. 121, 214-221 (2007).

${ }^{4}$ M. Q. Wu, "Micro-perforated panels for duct silencing," Noise Control Eng. J. 45, 69-77 (1997).
${ }^{5}$ D. Y. Maa, "Theory and design of microperforated panel sound absorbing construction" (in Chinese), Sci. Sin. 18, 55-71 (1975).

${ }^{6}$ D. Y. Maa, "Micorperforated Panel wide-band absorber," Noise Control Eng. J. 29, 77-84 (1987).

${ }^{7}$ D. Y. Maa, "Potential of microperforated panel absorber," J. Acoust. Soc. Am. 104, 2861-2866 (1998).

${ }^{8}$ X. Zha, J. Kang, T. Zhang, X. Zhou, and H. V. Fuchs, "Application approach for microperforated panel sound absorbers" (in Chinese), Acta Acustica 19, 258-265 (1994).

${ }^{9}$ M. Yairi, K. Takebayashi, K. Sakagami, and M. Morimoto, "Wideband sound absorber obtained by combination of two micro-perforated panel absorbers with different air cavity depths arranged in parallel," in Proceedings of inter-noise 2009, Ottawa, Canada, pp. 1-7, paper no. 341 (2009).

${ }^{10}$ K. Sakagami, Y. Nagayama, M. Morimoto, and M. Yairi, "Pilot study on wideband sound absorber obtained by combination of two different microperforated (MPP) absorbers," Acoustic. Sci. Tech. 30, 154-156 (2009).

${ }^{11}$ F. P. Mechel, "The wide-angle diffuser - a wide-angle absorber?" Acustica 81, 379-401 (1995).

${ }^{12}$ T. Wu, T. J. Cox, and Y. W. Lam, "From a profiled diffuser to an optimized absorber," J. Acoust. Soc. Am. 108, 643-650 (2000).

${ }^{13}$ J. P. Groby, W. Lauriks, and T. E. Vigran, "Total absorption peak by use of a rigid frame porous layer backed by a rigid multi-irregularities grating,” J. Acoust. Soc. Am. 127, 2865-2874 (2010).

${ }^{14}$ M. Yairi and K Sakagami, "Absorption characteristics of a microperforated panel absorber and increasing its absorption frequency range" (in Japanese), Proceedings of the Acoustical Society of Japan 2010 Spring Meeting, Tokyo, Japan, no. 1-4-11, pp. 1139-1142 (2010).

${ }^{15}$ C. Wang, L. Cheng, J. Pan, and G. Yu, "Sound absorption of a micro-perforated panel backed by an irregular-shaped cavity," J. Acoust. Soc. Am. 127, 238-246 (2010).

${ }^{16}$ ISO 10534-2, Determination of Sound Absorption Coefficient and Impedance in Impedance Tubes (International Organization for Standardization, Geneva, Switzerland, 1998).

${ }^{17}$ Y. Y. Lee, E. W. M. Lee, and C. F. Ng, "Sound absorption of a finite flexible micro-perforated panel backed by an air cavity," J. Sound Vib. 287, 227-243 (2005).

${ }^{18}$ M. Toyoda, R. L. Mu, and D. Takahashi, "Relationship between Helmholtz-resonance absorption and panel-type absorption in finite flexible microperforated-panel absorbers," Appl. Acoust. 71, 315-320 (2010).

${ }^{19}$ D. Takahashi and M. Tanaka, "Flexural vibration of perforated plates and porous elastic materials under acoustic loading," J. Acoust. Soc. Am. 112, 1456-1464 (2002).

${ }^{20}$ J. B. Keller and D. Givoli, "Exact non-reflecting boundary conditions," J. Comput. Phys. 82, 172-192 (1989). 